\title{
Research on Optimal Efficiency of Data in Pattern Design
}

\author{
Caisen HUANG*, Chunshan DENG, Li ZHOU \\ College of Textiles and Garments, Southwest University, Chongqing, China \\ DOI: $10.15221 / 17.078$ http://dx.doi.org/10.15221/17.078
}

\begin{abstract}
In the field of international manufacturing industry, innovative design and manufacturing technology for products have been developed from traditional labor technology to digitization and intellectualization. Whether it is emerging industry or traditional manufacturing industry, they have not been satisfied with the technical advantages provided by 2D technology and more 3D digital technologies have been integrated into them. With the advent of 3D era, 3D model construction, 3D digital imaging, 3D printing technology and so on have become a new impetus to promote product innovation and industry development.

Aiming at the situation that the research, about considering the optimal efficiency of the data in pattern design through the $3 \mathrm{D}$ manufacturing platform, is to be further improved, a research method to explore the optimal efficiency of the data form is proposed. Through tracing three-dimensional sizes of the human body, we selected the fifteen required body sizes of drawing pattern, which is designed with the help of the advantages of EMKO pattern and special human body measurement dimensions, from the data cluster. Moreover, we calculate the pattern type contrast score under different coverage ratio and carry on the optimal scale analysis and ROC curve fitting of the coverage ratio and pattern contrast score. Ultimately, according to the result of calculating and analyzing the coverage ratio and pattern contrast score and to the proof of experimental verification of 3D human body sample under the coverage ratio, the optimal circulation form of data in the process of pattern design can be determined.
\end{abstract}

Key words: Pattern design, Optimal efficiency, Coverage ratio, Curve fitting

With the advent of $3 \mathrm{D}$ era, we can quickly obtain 3D human data through the 3D body measuring instrument. It is particularly important for the development of garment industry to apply 3D human data to the actual production of the garment industry more scientifically, conveniently, accurately and quickly ${ }^{[1]}$. In recent years, the application of non-contact 3D scanning in apparel emerging, the trend from the manual measurement to the automation and digitizing has been increasingly obvious. As the needs of clothing of personalized customization continue to expand, the way producing garment through the principles of grading cannot meet the demand of users' personalization. To optimize this pattern, we decided to do a research on optimal efficiency of data in pattern design based on the relationship between body size and pattern.

\section{Background of the research}

In the process of pattern from standard to personality, some researchers put forward to do traditional grading through determining the key point, establishing the point number and modifying the rules ${ }^{[2-3]}$. And some other researchers came up with a kind of tailored system taking as the core the pattern generated rapidly according to users' $3 \mathrm{D}$ body data ${ }^{[4]}$. But these methods have some limitations, and have high requirements for graders.

Targeting such situation, as shown in Fig.1, we collected users' data accurately, verified strictly from comparison of prototype pattern to analysis of result, and achieve optimal efficiency of data in pattern design.

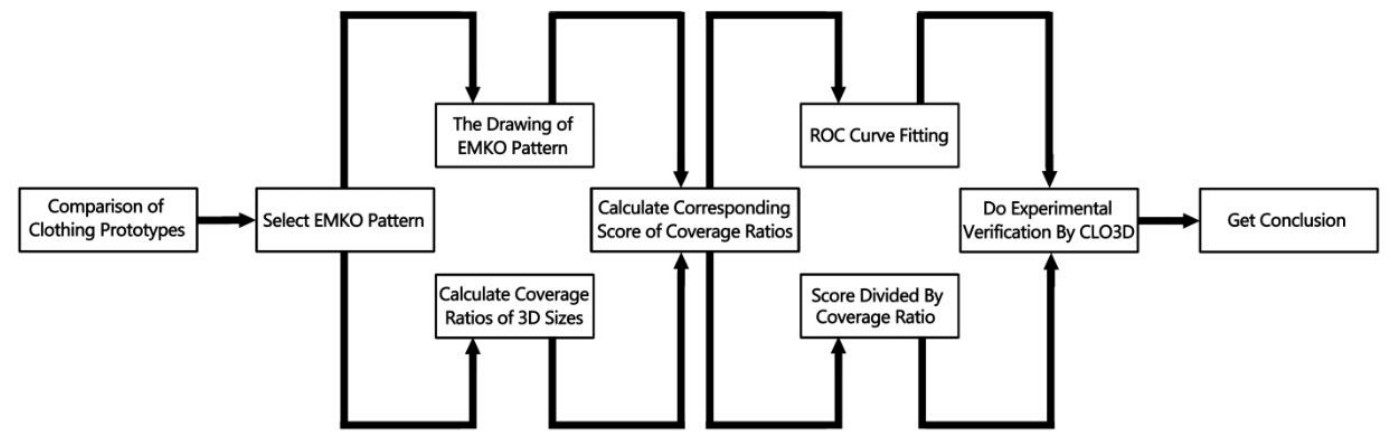

Fig. 1 Work flow of the research

\footnotetext{
* Fund project: This research is supported by "Fundamental Research Funds for the Central Universities (XDJK2016C100)". About the Author: HUANG Caisen, male, undergraduate, the main research direction is the digital clothing design. Communication author: ZHOU Li, E-mail: mydtcazz@126.com.
} 


\section{Process of the research}

\subsection{Select and draw the prototype object}

\subsubsection{Principle of selecting prototype object}

We need to fully consider the characteristics of human structure when taking measures of designing prototype pattern. In the meantime, we ought to adopt enough 3D body sizes in order to achieve personalized clothing customization ${ }^{[5]}$.

\subsubsection{Comparison of prototype patterns}

(1) American prototype: it looks more three-dimensional but it will not fit closely on the upper chest. And 22 body sizes are used in total.

(2) British prototype: the chest darts are on the shoulder seam separately. And it fit more closely as it possess traditional European prototype pattern's character and as vertical dividing lines are also closer to the lines of body. And 10 body sizes are used in total.

(3) Japanese prototype: based on consummate measurement data, it reduced body sizes to 4 according to the regression relationship among the measured data. Its structure has a close relationship with draping, and 4 body sizes are used in total.

(4) Donghua prototype: according to Chinese body size database, it was built based on Japanese prototype, and it is under adjusting and improving. And 4 body sizes are used in total.

Russia's EMKO prototype contains a large number of human body measurement sizes and geometric approximation mathematical operations, fully takes into account the characteristics of the human body structure, and adopts enough of the standard body sizes. As a consequence, this kind of prototype can be used in clothing customization, can also be widely used in industrial production. In order to highlight the characteristics of this research, we built the prototype pattern with the advantages of EMKO prototype design of and special human body measurement sizes

\subsubsection{Draw the prototype}

In the process of drawing the prototype, it comprised a total of 56 steps and involved the drawing of 50 points and of 6 parts of arc lengths. After simplification and statistics, a total of 15 body measurements were used in the entire drawing process. Due to space limitations, the steps of drawing are not described in detail here. And the result of prototype pattern design is shown in Fig.2.

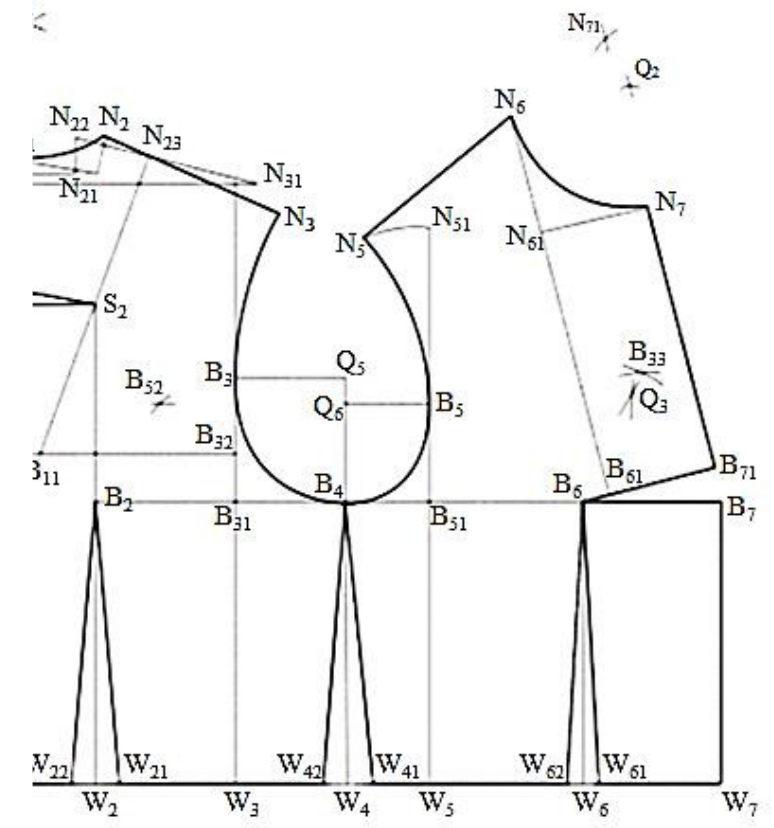

Fig.2 Drawing of the prototype pattern 


\subsection{Data analysis and verification of optimal efficiency}

\subsubsection{Calculation of score of comparison version and coverage}

In order to count the usage frequency and weight of 15 user data in drawing of the prototype, the raw data of the point and arc length were calculated and analyzed, and the results are shown in Table 1.

Tab.1 Data statistics of human body sizes

\begin{tabular}{cccc}
\hline Serial number & Location name & Usage frequency /times & Weights \\
\hline 1 & Chest height & 42 & $12.14 \%$ \\
2 & Distance between chest and waist & 42 & $12.14 \%$ \\
3 & Back across & 39 & $11.27 \%$ \\
4 & Nape to waist & 31 & $8.96 \%$ \\
5 & Armhole width & 31 & $8.96 \%$ \\
6 & Neck circumference & 30 & $8.66 \%$ \\
7 & Distance between back neck dot & 25 & $7.22 \%$ \\
8 & and line of back across & 24 & $6.96 \%$ \\
9 & Shoulder across & 20 & $5.77 \%$ \\
10 & Shoulder slope & 19 & $5.49 \%$ \\
11 & Back inclination & 18 & $5.20 \%$ \\
12 & Front inclination & 17 & $4.91 \%$ \\
13 & Chest across & 5 & $1.45 \%$ \\
14 & Bust girth & 2 & $0.58 \%$ \\
15 & Waist girth & 1 & $0.29 \%$ \\
\hline
\end{tabular}

Coverage calculation formula based on $n$ body $\operatorname{sizes}\left(n \in[1,15]\right.$ and $\left.n \in \mathrm{N}^{+}\right)$:

$$
\frac{n}{15} \times 100 \%
$$

According to the formula, we divided 15 body sizes at tab. 1 following by increments of coverage ratio. In the process of the drawing the prototype, body sizes that each unit involves covered a wide range, and it's difficult to correspond to coverage ratio and users' data, as a consequence, we replaced body sizes in Tab. 1 with $T_{1}-T_{15}$ in turn. Among 56 units (50 points and 6 parts of arc length) in the process of drawing the prototype, each unit was counted as 1 point and we can easily conclude that the version contrast score is 56 when the coverage ratio is $100 \%$. Assuming that $t$ was composed of polynomial relationship built by $a_{1} T_{1}, a_{2} T_{2} \ldots a_{14} T_{14}$ and $a_{15} T_{15}$, then the score of $T_{m}$ $(\{m \in N \mid 1 \leq m \leq 15\})$ in unit $t$ is:

$$
S^{t} T_{m}=\frac{1}{a_{1} T_{1}+a_{2} T_{2}+\cdots+a_{15} T_{15}}
$$

Then, among 56 units in the process of drawing the prototype, the total score of $T_{m}$ is:

$$
Q_{T_{m}}=\sum_{t=1}^{56} S^{t}{ }_{T_{m}}
$$

Therefore, we can calculate in turn the version contrast score in using $n\left(n \in[1,15]\right.$ and $\left.n \in \mathrm{N}^{+}\right)$body sizes:

$$
S_{n}=\sum_{m=1}^{n} Q_{T_{m}}=\sum_{m=1}^{n} \sum_{t=1}^{56} S^{t}{ }_{T_{m}}
$$

The result of calculating is shown as Tab.2. 
Tab.2 Coverage ratio classification results

\begin{tabular}{ccc}
\hline Body size (Serial number) & Coverage ratio & Version contrast score \\
\hline 1 & $6.67 \%$ & 6.51 \\
$1-2$ & $13.33 \%$ & 13.02 \\
$1-3$ & $20.00 \%$ & 20.26 \\
$1-4$ & $26.67 \%$ & 26.49 \\
$1-5$ & $33.33 \%$ & 33.44 \\
$1-6$ & $40.00 \%$ & 37.94 \\
$1-7$ & $46.67 \%$ & 41.08 \\
$1-8$ & $53.33 \%$ & 43.91 \\
$1-9$ & $60.00 \%$ & 46.74 \\
$1-10$ & $66.67 \%$ & 48.72 \\
$1-11$ & $73.33 \%$ & 50.49 \\
$1-12$ & $80.00 \%$ & 52.43 \\
$1-13$ & $86.67 \%$ & 55.13 \\
$1-14$ & $93.33 \%$ & 55.56 \\
$1-15$ & $100.00 \%$ & 56.00 \\
\hline
\end{tabular}

[Note: the line named with "Body size(Serial number)" in Tab.2 follows the line named with "Serial number" in Tab.1. For example, "1-3" in Tab. 2 represent the body sizes that " $1,2,3$ " correspond to in Tab.1.]

\subsubsection{ROC curve fitting and optimal scale regression}

As we could not observe the relationship obviously between coverage ratio and the version contrast score according to data listed in Tab.2, the scatter plot was drawn in accordance with Tab.2 and the result is shown as Fig.3. Then we finished ROC curve fitting based on the scatter plot, as is shown in Fig.4.

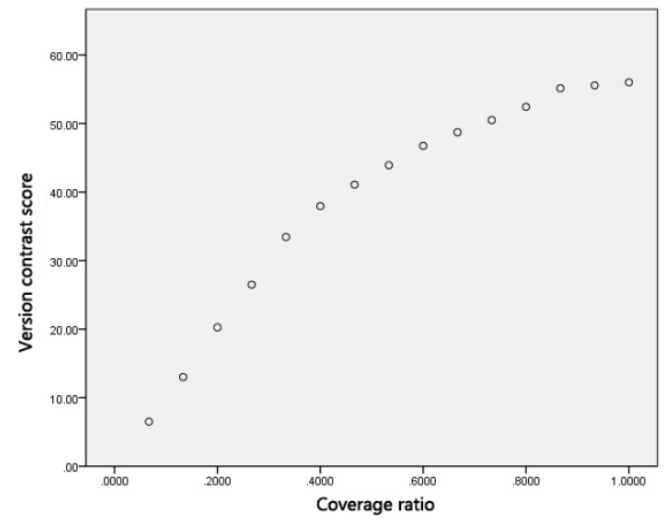

Fig.3 The coverage ratio and version contrast score scatter gram

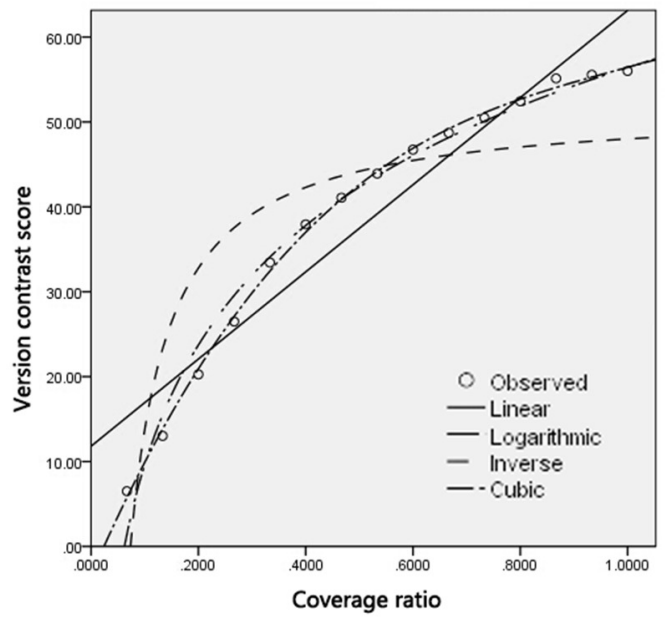

Fig.4 The coverage ratio and version contrast score curve fitting graph 
We did ROC curve fitting according to Fig. 3 with the help of SPSS 19.0 data statistical analysis software. According to the trend shown in the scatter plot, we finished 4 types fitting of function including Linear, Inverse, Cubic and Logarithmic at the same time, as is shown in Fig.4.

The degree of fitting of 4 types of function to the data is not the same, which reflected in $R$ square and variance to some degree. And the result is shown in Tab.3.

Tab.3 Comparison result of functions

\begin{tabular}{ccc}
\hline Type of function & $R$ square & Variance \\
\hline Linear & 0.982 & 786.698 \\
Inverse & 0.998 & 2149.721 \\
Cubic & 0.75 & 42.966 \\
Logarithmic & 0.907 & 137.648 \\
\hline
\end{tabular}

Statistical results in Tab.3 show that $R$ square and variance of the cubic function are minimal and it can also be seen from Fig. 4 that the degree of fitting of the cubic function is optimal.

As the version contrast score corresponds to the coverage ratio one to one and as they are sets of points, it's not suitable to do optimal calculation through calculating the derivative function for fitting parabolic function model. By calculating and observing the ratio of 15 groups of the coverage ratio and version contrast score, we examined through parabolic function and calculated the optimal result. In order to ensure the rationality of data collection and the necessity of this study, the ratio greater than or equal to $80 \%$ and less than or equal to $20 \%$ should be excluded, as shown in Tab.4.

Tab.4 Ratio calculation result

\begin{tabular}{ccc}
\hline Body size (Serial number) & Coverage ratio & Version contrast score/ Coverage ratio \\
\hline $1-4$ & $26.67 \%$ & 99.32 \\
$1-5$ & $33.33 \%$ & 100.33 \\
$1-6$ & $40.00 \%$ & 94.84 \\
$1-7$ & $46.67 \%$ & 88.03 \\
$1-8$ & $53.33 \%$ & 82.33 \\
$1-9$ & $60.00 \%$ & 77.89 \\
$1-10$ & $66.67 \%$ & 73.08 \\
$1-11$ & $73.33 \%$ & 68.85 \\
\hline
\end{tabular}

As can be seen from the table above, the ratio of the version contrast score to the coverage ratio is the highest when the coverage ratio is $33.33 \%$. That is, data transpose efficiency in drawing prototype is optimal.

\subsection{3. $3 D$ operation platform experiment}

We drew a comparison about the fitting of prototype (as shown in Fig.2) between 100\% and $33.33 \%$ coverage ratio with the help of "CLO3D 2011". And the result is shown in Fig.5 and Fig.6.

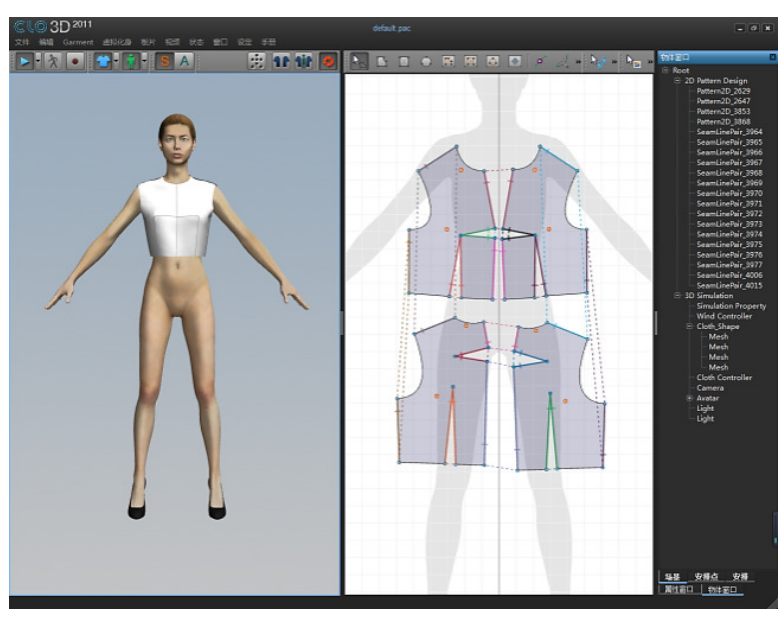

Fig.5 3D virtual fitting under $33.33 \%$ coverage ratio

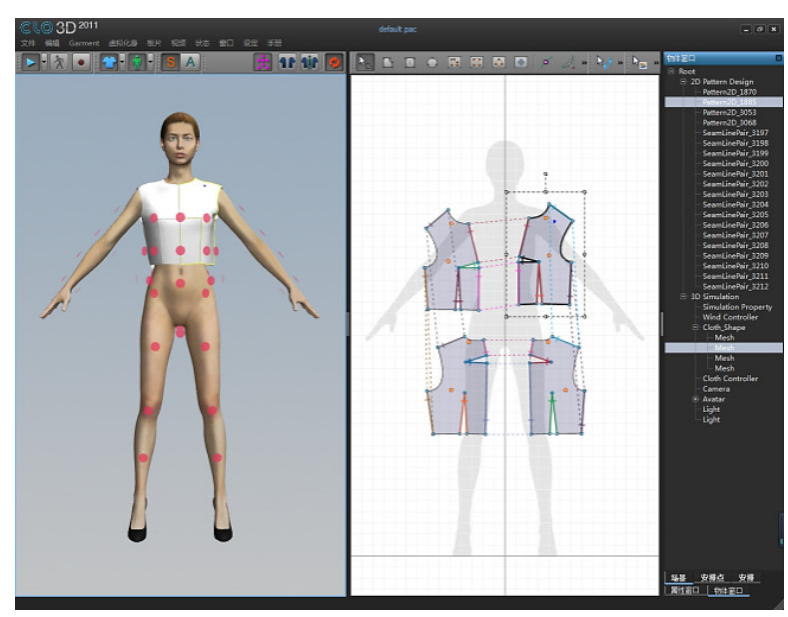

Fig.6 3D virtual fitting under $100 \%$ coverage ratio 
As long as the process data associated with chest height, distance between chest and waist, back across, nape to waist and armhole width are determined in $33.33 \%$ coverage ratio, we can provide users with more efficient customization through adjusting other data. And it is more likely to fit the different characteristics of user base compared to the prototype with $100 \%$ coverage ratio.

\subsection{Conclusion}

Taking as the research object the prototype built with the some advantage of EMKO prototype and with special body size measurements, we extracted data needed in the process of drawing the prototype. After data processing and ROC curve fitting, the fitting results show that efficiency is optimal when the coverage ratio is $33.33 \%$, in the meanwhile, the corresponding user data are chest height, distance between chest and waist, back across, nape to waist and armhole width. Then we did verification about the coverage ratio under the optimal efficiency with the help of CLO3D, and we concluded that $33.33 \%$ is the optimal considering fitness degree and efficiency. That is to say, except the data corresponded to $33.33 \%$ coverage ratio, other data should be carried out manual adjustment according to figure. Therefore, body data put into production should be reduced to chest height, distance between chest and waist, back across, nape to waist and armhole width when selecting the prototype created in this article as the base prototype. By the way, collection of users' data varies from prototype to prototype, but the research methods in this paper are generally applicable.

\section{References}

[1] WEN Wu. The anthropometric data based on three dimensional clothing structural design applied research [J]. Tianjin: Tianjin University Of Science \& Technology, 2010:9-10

[2] Cynthia L, Istook. Enabling mass customization: computer-driven alteration methods [J], International Journal of Clothing Science and Technology, 2002, 14(1): 61-76

[3] YANG Yunchu, ZHANG Weiyuan, LIU Li. Exploring of digital garment pattern design based on mass customization[J]. Journal of Textile Research, 2007, 28(1): 124-128.

[4] HU Jueliang, DONG Jianming, HE Ying, et al. Garment pattern design based on artificial neural network [J]. Journal Of Textile Research, 2010, 2006, 27(2): 49-52.

[5] Qi Xingxiang. Research on evaluation model of clothes fit based on individual virtual mannequin [D]. Shanghai: Donghua University, 2011: 4-7. 